\title{
Erupción y Retención del Tercer Molar en Jóvenes entre 17 y 20 Años, Antofagasta, Chile
}

\author{
Eruption and Retention of Third Molars in Young People \\ between 17 and 20 Years of Age, Antofagasta, Chile
}

"Fernando García-Hernández; "** Oscar Toro Yagui; "**Manuel Vega Vidal \& **Mauricio Verdejo Meneses

GARCÍA-HERNÁNDEZ, F.; TORO, Y. O.; VEGA, V. M. \& VERDEJO, M. M. Erupción y retención del tercer molar en jóvenes entre 17 y 20 años, Antofagasta, Chile. Int. J. Morphol., 27(3):727-736, 2009.

RESUMEN: La retención intraósea y la erupción en malposición de 3Ms han sido muy estudiadas. Son causadas, probablemente, por disminución del tamaño de maxilares por cambios de hábitos alimentarios, reduciendo el espacio retromolar, lo que dificulta la erupción normal entre 15 y 25 años de edad, y produce patologías o molestias por comprometer estructuras orofaciales próximas. Lo anterior, ha promovido la exodoncia profiláctica u ortodóncica, incluso del germen dentario, con altos costos clínicos, hospitalarios, laborales, comprometiendo parte de los recursos destinados a financiar otros procedimientos quirúrgicos orales, además de riesgos durante cirugía, postoperatorio y lesiones iatrogénicas temporales o permanentes. La muestra consistió en 100 jóvenes de 17 a 20 años de edad (50 mujeres y 50 hombres) de la ciudad de Antofagasta, sanos, sin malformaciones general y maxilofacial, sin haber presentado enfermedades infecciosas que alteraran odontogénesis y períodos eruptivos, sin exodoncias de $3 \mathrm{M}$ ni tratamientos ortodóncicos previos al examen de la radiografía panorámica y clasificando erupción de 3Ms según tablas de Pell-Gregory y Winter. Se determina 49,1\% de $3 \mathrm{Ms}$ retenidos con $\mathrm{p}<0,05$ significativo respecto dientes erupcionados, predominando retención maxilar, especialmente en mujeres. En todos los casos y en mandíbula predominan $3 \mathrm{Ms}$ con impactación mesioangulada ( $<<0,05$ significativa) y en maxilares la retención vertical ( $<<0,05$ de significancia). Prevalecen 3Ms distoangulados en maxilares, posición que predispone a complicaciones operatorias y postoperatorias en exodoncias. La retención horizontal se aprecia en mandibula, siendo el segundo tipo de retención (21,5\% en toda la muestra y $30 \%$ en hombres). Sin una decisión clínica que indique la cirugía, se sugiere postegar la exodoncia profiláctica de $3 \mathrm{Ms}$, esperando posible erupción tardía (Hattab, 1997; Ventä et al. 1999, 2004 y Kruger et al.). Meta-análisis demuestra: mínima morbilidad en exodoncias de $3 \mathrm{Ms}$ en pacientes de 25 años o mayores, baja incidencia de complicaciones y mínimo impacto en calidad de vida (Haug et al., 2005).

PALABRAS CLAVE: Tercer molar; Retención; Clasificación de Pell-Gregory; Clasificación de Winter; Odontología.

\section{INTRODUCCIÓN}

Es conocida la teoría de la reducción terminal dentaria de Adloff (citado por Figún \& Garino, 1992) que señala la futura desaparición del $3 \mathrm{M}$ en la especie humana, aspecto evolutivo consecuencia de la disminución de la actividad masticatoria. Esta sería una línea evolutiva hacia un número menor de dientes (Shafer et al., 1977). En 2001, Proffit, citado por Arboleda et al. (2006), indica que las tendencias evolutivas han influido en la dentición actual y en la dimensión de los maxilares, en tanto que Bailit (1975) sugiere que la fórmula dentaria futura podría excluir a incisivos laterales, segundos premolares y terceros molares. Björk (1956) señala que el $45 \%$ de la población tendrá retención de uno o más $3 \mathrm{Ms}$. Recientemente el examen radiográfico digital de la mandíbula de la "niña Magdaleniense", de 13.000-15.000 años de antigüedad, muestra un $3 \mathrm{M}$ retenido en posición mesioangular, considerado el caso más antiguo reconocido de impactación del 3M (Flygare \& Öhman, 2008).

Según señalan Figún \& Garino, la evolución de los hábitos alimenticios ha contribuido a modificar el tamaño de los maxilares, siendo marcado este cambio a nivel mandibular, aspecto evolutivo del Homo sapiens sapiens que

\footnotetext{
* Unidad de Anatomía, Departamento Biomédico, Facultad de Ciencias de la Salud, Universidad de Antofagasta, Antofagasta, Chile.

** Cirujano Dentista, Clínica de Ortodoncia Manquehue, Antofagasta, Chile.
} 
se confirma por la disminución progresiva del espacio retromolar entre borde anterior de la rama y cara distal del 3M (Fig. 1), lo que actualmente se determina mediante la clasificación de Pell \& Gregory (Fig. 2), que mide el espacio que tiene el $3 \mathrm{M}$ para erupcionar entre borde anterior de rama y cara distal del 2M (Sandhu \& Kaur, 2005).
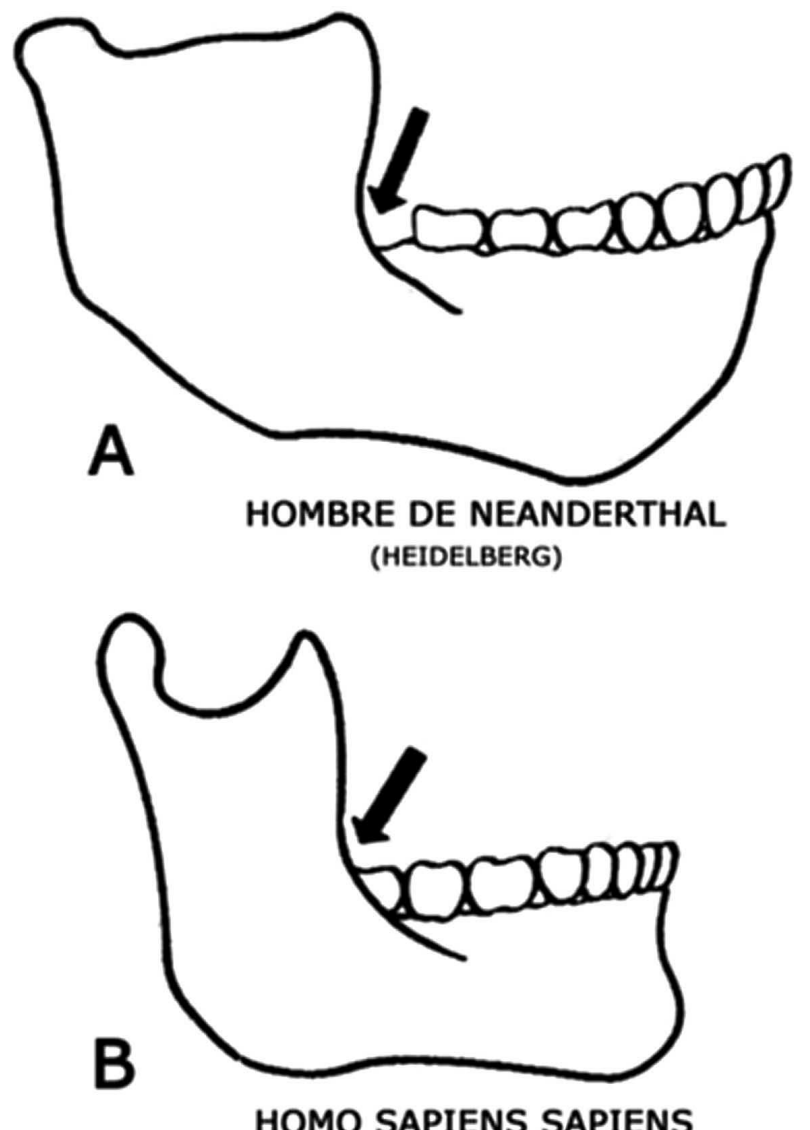

Fig. 1. Disminución del espacio retromolar por variaciones de la forma y tamaño mandibulares producto de la evolución y de los cambios de hábitos masticatorios (adaptado y modificado de Figún \& Garino).

Un punto de vista que apoya la teoría de la evolución, se basa en la presunción que los maxilares han ido disminuyendo su tamaño durante la evolución humana, probablemente como resultado de una reducción evolutiva en el tamaño corporal genéticamente determinado, concepto que justifica la gran incidencia de agenesia de 3Ms inferiores (Anderson et al., citado por Sarmiento \& Herrera, 2004).

Kruger, en 1984 (citado por Nicodemo Fhilo et al., 2008), señala que una dieta más refinada, requiriendo menos masticación, ocasiona esta tendencia, haciendo menos necesario un aparato masticatorio potente. Pos estas y otras razones, un número cada vez mayor de personas presenta inclusiones dentarias y agenesias.

Hattab \& Alhaija (1999) señalan que la menor exigencia masticatoria a consecuencia de los hábitos alimenticios actuales disminuye el tamaño de los maxilares y dificulta la erupción del 3M, favoreciendo la retención.

Confirma las hipótesis anteriores, la investigación realizada en adolescentes nigerianos que habitaban en el medio rural y que a los 19 años de edad tenían erupcionados todos los $3 \mathrm{M}$, sin agenesia y sin retenciones y que por necesidades alimentarias, debían utilizar en forma enérgica su aparato masticatorio (Odusanya \& Abayomi, 1991).

Los aumentos de la agenesia y de la retención del $3 \mathrm{M}$ ha ido de la mano con la evolucion humana. Nicodemo Filho et al. (2008) destacan la opinión de antropólogos que afirman que el crecimiento constante del cerebro aumenta el volumen de la cavidad craneana en desmedro de los maxilares, lo que contribuye a que un número cada vez mayor de personas presenten alteraciones en la erupción normal de 3Ms.

La revisión de literatura establece un amplio rango de $3 \mathrm{M}$ no erupcionados, desde $22,3 \%$ planteado por Björk hasta 66,6\% determinado por Vënta et al., (1991); en cambio, como fue señalado anteriormente, Odusanya \& Abayomi comprueban $100 \%$ de erupción en nigerianos. Silvestri \& Sing (2003) hacen referencia que un 65\% de la población humana de 20 años de edad ha tenido, a lo menos, un $3 \mathrm{M}$ impactado.

El período normal de erupción del 3M (entre los 17 y 25 años de edad) y la mayor calcificación de los maxilares que complica dicha erupción, la malposición post erupción de dichas piezas dentarias con severas alteraciones de la oclusión y apiñamiento dentario, el aumento del acceso a atención odontológica de especialistas odontopediatras y ortodoncistas, la información transmitida como referencia parental señalando las molestias y tratamientos sufridos durante el largo período eruptivo, así como, un amplio abanico comunicacional que permite acceder a mucha información al respecto, han provocado un aumento de la exodoncia profiláctica y de la recomendación ortodóncica de exodoncia (Olate et al. 2007).

Friedman $(1983,2007)$ indica al respecto, que pese a que $3 \mathrm{Ms}$ producen condiciones que justifican su exodoncia, se ha visto que estos dientes son removidos rutinariamente para prevenir serios malestares que no tienen soporte científico. Impactación es el término usado más frecuentemente 

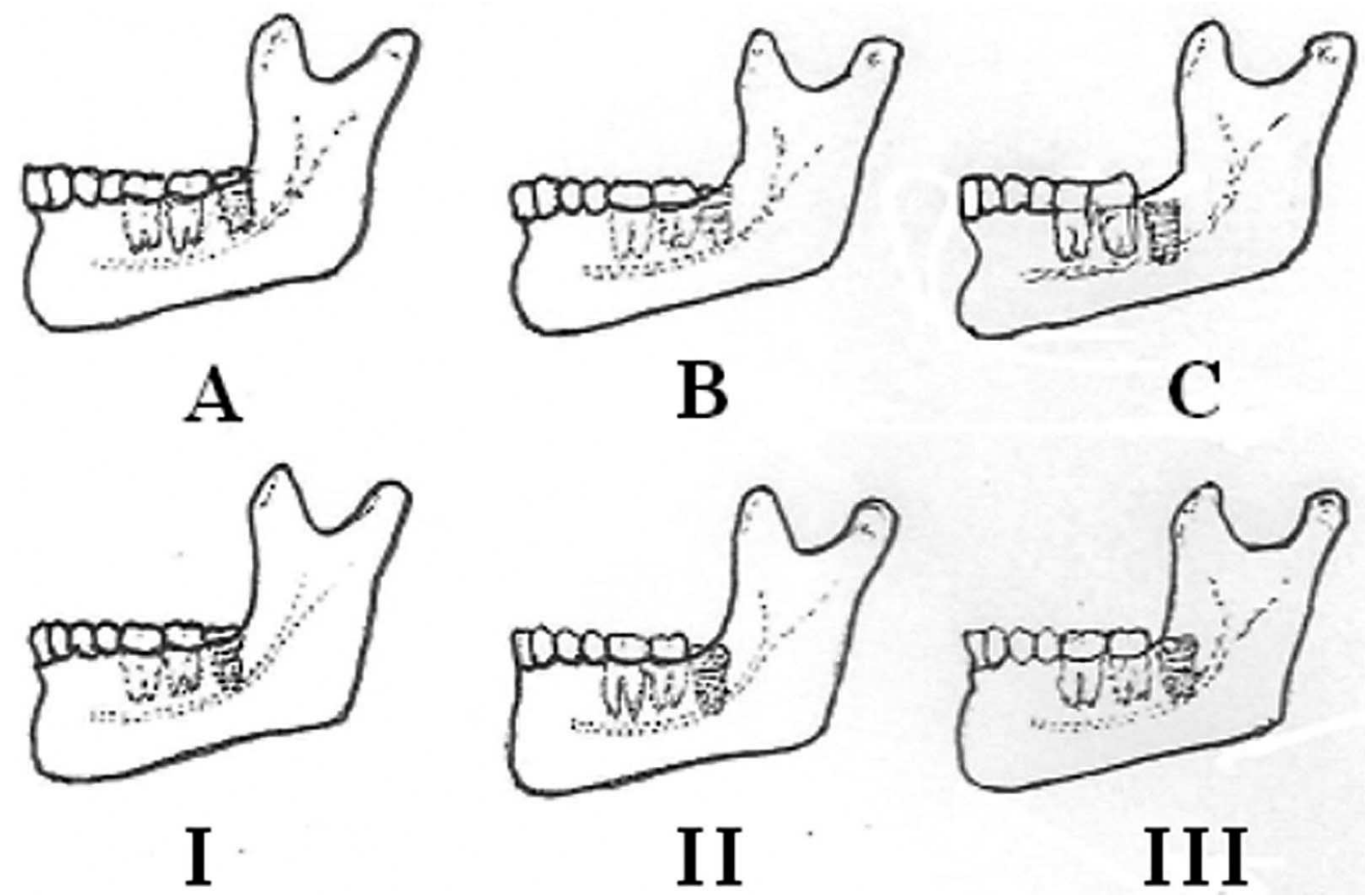

Fig 2. Clasificación de Pell-Gregory sobre nivel de erupción de tercer molar mandibular. A= erupcionado, B= semierupcionado, $\mathrm{C}=$ retenido, $\mathrm{I}=$ adecuado espacio retromolar, $\mathrm{II}=$ espacio retromolar insuficiente, $\mathrm{III}=$ tercer molar parcial o totalmente dentro de la rama mandibular (Adaptado y modificado de Halmos et al., 2004).

para describir un proceso quirúrgico más que un diagnóstico clínico. Señala que el alto costo acumulativo de las cirugías del 3M excede los costos de cualquier otro procedimiento quirúrgico y recomienda restringir el pago de seguros para cirugías profilácticas del $3 \mathrm{M}$, lo que liberaría grandes sumas de dinero o recursos económicos que podrían ser usados en pacientes con reales enfermedades dentales. Susarla \& Dodson (2005), señalan que los costos clínicos de la exodoncia del 3M irrogan el 50\% de todos los procedimientos quirúrgicos orales.

En este mismo sentido, Silvestri \& Sing se refieren al poco valor funcional que se otorga al $3 \mathrm{M}$ y que, asociado a una alta tasa de dolor y malestar que producen durante el período eruptivo ha estimulado los procedimientos quirúrgicos, con alto costo por tiempo laboral perdido, radiografías, medicación, anestesia o costos hospitalarios, pérdida de ingresos por licencias, etc. Friedman reitera que el procedimiento quirúrgico no está exento de riesgos, tales como parestesia, daños iatrogénicos como fractura mandibular, compromiso de ATM, seno maxilar, tuberosidad del maxi- lar, dientes vecinos y hasta la muerte por causa de la anestesia general. Además, no debe olvidarse que los $3 \mathrm{Ms}$ pueden ser muy importantes con fines protésicos, cuando se han perdido otros dientes.

Siendo el 3M el diente que provoca mayores patologías asociadas con su presencia, retención, erupción, variabilidad de posición una vez erupcionado y cuyo síntoma principal es el dolor que produce, así como las dificultades quirúrgicas durante su exodoncia tales como el ya señalado riesgo de fractura mandibular y otras iatrogenias (Halmos $e t$ al.) y las complicaciones postquirúrgicas que conlleva (2,27\% de los pacientes atendidos en el año 2006, en la red del Ministerio de Salud de Chile presentaron reacciones adversas producto de la extracción del 3M, Ministerio de Salud de Chile, 2008), así como los importantes aspectos económicos involucrados en tratamiento por ausencias laborales y licencias médicas pre y postquirúrgicas (Friedman, 1983, 2007; Cauvi \& Feldman, 1989; Ahlqwist \& Gröndahl, 1991; Feldman et al., 1995; Martínez et al., 1995; Muhonen et al., 1997; Punwutikorn et al., 1999; Silvestri \& Sing; Cha- 
parro-Avendaño et al., 2005), es que efectuamos este estudio radiológico para determinar el grado de erupción y situación de retención de $3 \mathrm{Ms}$ en una muestra de jóvenes de 17 a 20 años de edad que habitan la ciudad de Antofagasta, como un aporte a la anatomía odontológia y poder efectuar comparaciones de los resultados con los obtenidos por otros investigadores.

\section{MATERIAL Y MÉTODO}

Se revisaron alrededor de 3.000 fichas clínicas desde el archivo de Clínica de Ortodoncia Manquehue de Antofagasta, Chile, eligiendo 100 casos de individuos jóvenes, entre 17 y 20 años de edad, con una edad promedio de 18 años y 6 meses de ambos géneros ( 50 hombres y 50 mujeres). Tabla I.

Las radiografías panorámicas debían corresponder a la primera radiografía diagnóstica de pacientes sanos, sin ningún tipo de malformaciones general y maxilofacial, que no hubiesen presentado enfermedades infecciosas que alteraran la odontogénesis y los períodos de erupción, sin exodoncias de ningún 3M y sin tratamiento ortodóncico previo al examen radiográfico.

De los casos seleccionados se registraron los siguientes datos: Nombre, edad, fecha de nacimiento y del examen radiográfico, historia clínica relevante, presencia de agenesias, grado de formación (clasificación de Nolla) y nivel de erupción del 3M (clasificaciones de Pell \& Gregory (1933) y de Winter (1926), Fig. 2 y Tabla II), antecedentes que fueron vertidos a un protocolo de investigación, tomando registro fotográfico de cada radiografía panorámica. Tabla II.

Los datos fueron tabulados en Microsoft Excel. Para el análisis estadístico se empleó programa Statgraphics Plus 5.1

\section{RESULTADOS}

Los resultados obtenidos del análisis estadístico determinado para la muestra de jóvenes de 17 a 20 años de edad de la ciudad de Antofagasta, Chile, se detallan en las Tablas siguientes (Tablas III a VIII).

\section{DISCUSIÓN}

La muestra consistió en 100 individuos (50 mujeres y 50 hombres) con rangos de edad entre 17 y 20 años y con una edad promedio de 18 años y 6 meses (Tabla I).

En el grupo en estudio se determinó un $32 \%$ de casos con agenesia de uno o más 3Ms (Tabla V), porcentaje más alto que los obtenidos por el autor y colaboradores en otros trabajos realizados en el Norte de Chile, pero sin diferencia estadística significativa al 95\% (García-Hernández \& Beltrán, 2008=26,7\% p<0,423741; García-Hernández et al., 2008=24,75\% p<0,140292; García-Hernández \& Beltrán, 2009=21,79\% p<0,130225; García-Hernández \& Araneda, $2009=20,0 \% \mathrm{p}<0,0530541)$.

Tabla I. Características de la muestra.

\begin{tabular}{lccccc}
\hline \multicolumn{1}{c}{ Género } & n & Edad & Rango edad & \% \\
\hline Masculino & 50 & 18 años 7 meses & 17 años-20 años 11 meses & 50,0 \\
Femenino & 50 & 18 años 5 meses & 17 años-20 años 11 meses & 50,0 \\
\hline Total & 100 & X 18 años 6 meses & 17 años-20 años 11 meses & 100,0 \\
\hline
\end{tabular}

Tabla II. Clasificaciones de grados o niveles de erupción de terceros molares.

\begin{tabular}{|c|c|c|}
\hline \multicolumn{2}{|c|}{ Clasificación de Pell-Gregory } & \multirow[b]{2}{*}{$\begin{array}{l}\text { Winter } \\
\text { Tipos de } 3^{\text {os }} \text { molares en } \\
\text { relación a su posición respecto } \\
\text { eje longitudinal del } 2^{\circ} \text { molar: } \\
\text { - Vertical }\end{array}$} \\
\hline $\begin{array}{l}\text { - Relación del } 3^{\text {er }} \text { molar con rama mandibular: } \\
\text { Clase I=Espacio entre la superficie distal del } \\
2^{\circ} \text { molar y rama es mayor que diámetro } \\
\text { mesiodistal del } 3^{\text {er }} \text { molar. }\end{array}$ & $\begin{array}{l}\text { - Profundidad relativa del } 3^{\text {er }} \text { molar: } \\
\text { Posición A= La parte más al ta del } 3^{\text {er }} \text { molar } \\
\text { está al mismo nivel o por e ncima del plano } \\
\text { oclusal del } 2^{\circ} \text { molar. }\end{array}$ & \\
\hline $\begin{array}{l}\text { Clase II= Espacio entre la superficie distal } \\
\text { del } 2^{\circ} \text { molar y rama es menor que diámetro } \\
\text { mesiodistal del } 3^{\text {er }} \text { molar. }\end{array}$ & $\begin{array}{l}\text { Posición } B=\text { La parte más alta del } 3^{\text {er }} \text { molar } \\
\text { está entre la línea oclusal y el cuello del } 2^{\circ} \\
\text { molar. }\end{array}$ & $\begin{array}{l}\text { - Mesia angulado } \\
\text { - Disto angulado } \\
\text { - Horizontal }\end{array}$ \\
\hline $\begin{array}{l}\text { Clase } \mathrm{III}=\mathrm{El} 3^{\mathrm{er}} \text { molar está parcial o } \\
\text { totalmente dentro de la rama mandibular. }\end{array}$ & $\begin{array}{l}\text { Posición C }=\text { La parte más alta del } 3^{\text {er }} \text { molar } \\
\text { está al mismo nivel o por debajo del cuello } \\
\text { del } 2^{\circ} \text { molar. }\end{array}$ & $\begin{array}{l}\text { - Vestíbulo versión } \\
\text { - Linguo versión } \\
\text { - I nvertido }\end{array}$ \\
\hline
\end{tabular}


Tabla III. Clasificación Pell-Gregory. Erupción de terceros molares en relación a profundidad relativa (maxila-mandíbula) y relación con la rama mandibular en terceros molares inferiores. Mujeres y hombres de 17-20 años de edad, Antofagasta, Chile ( $\mathrm{n}=50)$.

\begin{tabular}{|c|c|c|c|c|c|c|c|c|c|c|c|}
\hline \multicolumn{6}{|c|}{ Mujeres $n=165$ terceros molares } & \multicolumn{6}{|c|}{ Hombres $n=167$ terceros molares } \\
\hline \multirow{2}{*}{$\begin{array}{l}\text { Maxila } \\
1.8 \\
\end{array}$} & \multirow{2}{*}{$\begin{array}{r}\mathrm{n}=83 \\
2.8\end{array}$} & \multicolumn{4}{|c|}{ Mandíbula $\mathrm{n}=82$} & \multirow{3}{*}{$\begin{array}{c}\text { Maxi } \\
1.8 \\
\text { Nivel }\end{array}$} & \multirow{3}{*}{$\begin{array}{c}\mathrm{n}=85 \\
2.8 \\
\text { Nivel }\end{array}$} & \multicolumn{4}{|c|}{ Mandíbula $\mathrm{n}=82$} \\
\hline & & \multicolumn{2}{|c|}{3.8} & \multicolumn{2}{|c|}{4.8} & & & \multicolumn{2}{|c|}{3.8} & \multicolumn{2}{|c|}{4.8} \\
\hline Nivel & Nivel & Clase & Nivel & Clase & Nivel & & & Clase & Nivel & Clase & Nivel \\
\hline$A=8$ & $A=9$ & $\mathrm{I}=25$ & $A=8$ & $\mathrm{I}=28$ & $A=9$ & $A=10$ & $A=12$ & $\mathrm{I}=32$ & $A=10$ & $\mathrm{I}=29$ & $A=11$ \\
\hline $18,6 \%$ & $22,5 \%$ & $64,1 \%$ & $20,5 \%$ & $65,1 \%$ & $20,9 \%$ & $23,8 \%$ & $27,9 \%$ & $74,4 \%$ & $20,5 \%$ & $74,4 \%$ & $28,2 \%$ \\
\hline $\mathrm{B}=11$ & $B=11$ & $\mathrm{II}=2$ & $B=16$ & $\mathrm{II}=2$ & $B=14$ & $B=10$ & $B=13$ & $\mathrm{II}=1$ & $B=19$ & $\mathrm{II}=4$ & $B=14$ \\
\hline $25,6 \%$ & $27,5 \%$ & $5,1 \%$ & $41 \%$ & $4,7 \%$ & $32,6 \%$ & $23,8 \%$ & $30,2 \%$ & $2,3 \%$ & $41 \%$ & $10,2 \%$ & $35,9 \%$ \\
\hline$C=24$ & $\mathrm{C}=20$ & $\mathrm{III}=12$ & $\mathrm{C}=15$ & $\mathrm{III}=13$ & $\mathrm{C}=20$ & $\mathrm{C}=22$ & $\mathrm{C}=18$ & $\mathrm{III}=10$ & $\mathrm{C}=14$ & $\mathrm{III}=6$ & $\mathrm{C}=14$ \\
\hline $55,8 \%$ & $50,0 \%$ & $30,8 \%$ & $38,5 \%$ & $30,2 \%$ & $46,5 \%$ & $52,4 \%$ & $41,9 \%$ & $23,3 \%$ & $38,5 \%$ & $15,4 \%$ & $35,9 \%$ \\
\hline
\end{tabular}

Tabla IV. Clasificación de Winter. Nivel de erupción del tercer molar respecto de la cara distal del segundo molar. Mujeres, hombres y total de muestra de jóvenes de 17-20 años de edad, Antofagasta, Chile (n=100).

\begin{tabular}{|c|c|c|c|c|c|c|c|c|c|c|c|c|}
\hline \multirow{2}{*}{$\frac{\text { Clasificación Winter }}{\text { Posición }}$} & \multicolumn{4}{|c|}{ Mujeres $\mathbf{n = 5 0}$} & \multicolumn{4}{|c|}{ Hombres $n=50$} & \multicolumn{4}{|c|}{ Total muestra $n=100$} \\
\hline & 1.8 & 2.8 & 3.8 & 4.8 & 1.8 & 2.8 & 3.8 & 4.8 & 1.8 & 2.8 & 3.8 & 4.8 \\
\hline Vertical & 27 & 28 & 16 & 16 & 34 & 33 & 11 & 15 & 61 & 33 & 11 & 15 \\
\hline & $62,8 \%$ & $70 \%$ & $41 \%$ & $37,2 \%$ & $81 \%$ & $76,7 \%$ & $25,6 \%$ & $38,4 \%$ & $\%$ & $76,7 \%$ & $25,6 \%$ & $38,4 \%$ \\
\hline Mesio angulado & $\begin{array}{c}10 \\
23,3 \%\end{array}$ & $\begin{array}{c}5 \\
12,5 \%\end{array}$ & $\begin{array}{c}16 \\
41 \%\end{array}$ & $\begin{array}{c}21 \\
48,8 \%\end{array}$ & $\begin{array}{c}4 \\
9,5 \%\end{array}$ & $\begin{array}{c}3 \\
7 \%\end{array}$ & $\begin{array}{c}20 \\
46,5 \%\end{array}$ & $\begin{array}{c}19 \\
48,7 \%\end{array}$ & $\begin{array}{l}14 \\
\%\end{array}$ & $\begin{array}{c}3 \\
7 \%\end{array}$ & $\begin{array}{c}20 \\
46,5 \%\end{array}$ & $\begin{array}{c}19 \\
48,7 \%\end{array}$ \\
\hline Disto angulado & $\begin{array}{c}6 \\
13,9 \%\end{array}$ & $\begin{array}{c}7 \\
16,3 \%\end{array}$ & $\begin{array}{c}1 \\
2,6 \%\end{array}$ & 0 & $\begin{array}{c}4 \\
9,5 \%\end{array}$ & $\begin{array}{c}7 \\
16,3 \%\end{array}$ & $\begin{array}{c}3 \\
7 \%\end{array}$ & $\begin{array}{c}1 \\
2,6 \%\end{array}$ & $\begin{array}{l}10 \\
\%\end{array}$ & $\begin{array}{c}7 \\
16,3 \%\end{array}$ & $\begin{array}{c}3 \\
7 \%\end{array}$ & $\begin{array}{c}1 \\
2,6 \%\end{array}$ \\
\hline Horizontal & & & $\begin{array}{c}4 \\
10,3 \%\end{array}$ & $\begin{array}{c}1 \\
2,4 \%\end{array}$ & & & $\begin{array}{c}8 \\
18,6 \%\end{array}$ & $\begin{array}{c}4 \\
10,3 \%\end{array}$ & & & $\begin{array}{c}8 \\
18,6 \%\end{array}$ & $\begin{array}{c}4 \\
10,3 \%\end{array}$ \\
\hline Vestíbulo lingual & & & $\begin{array}{c}2 \\
5,1 \%\end{array}$ & $\begin{array}{c}5 \\
11,6 \%\end{array}$ & & & $\begin{array}{c}1 \\
2,3 \%\end{array}$ & 0 & & & $\begin{array}{c}1 \\
2,3 \%\end{array}$ & 0 \\
\hline
\end{tabular}

Tabla V. Erupción, agenesia y retención de terceros molares. Mujeres, hombres y total de muestra de jóvenes de 17-20 años de edad, Antofagasta, Chile $(\mathrm{n}=100)$. 1) $\mathrm{p}<0,185428$, sin diferencia estadística significativa al $95 \%$ entre retención de terceros molares entre ambas arcadas en mujeres. 2) p <0,825991, sin diferencia estadística significativa al $95 \%$ entre retención de terceros molares entre ambas arcadas en hombres. 3) p <0,742905, sin diferencia estadística significativa al $95 \%$ entre retención de terceros molares entre ambas arcadas en la totalidad de la muestra. 4)p $<0,000123822$, con diferencia estadística significativa al $95 \%$ entre terceros molares erupcionados y retenidos en la totalidad de la muestra.

\begin{tabular}{|c|c|c|c|c|c|c|c|}
\hline \multirow{2}{*}{$\begin{array}{l}\text { Nivel de posición } \\
\text { eruptiva del tercer } \\
\text { molar. Clasificación } \\
\text { de Pell-Gregory }\end{array}$} & \multicolumn{2}{|c|}{ Mujeres $n=165$ terceros molares } & \multicolumn{2}{|c|}{$\begin{array}{c}\text { Hombres } n=167 \text { terceros } \\
\text { molares }\end{array}$} & \multicolumn{2}{|c|}{$\begin{array}{c}\text { Totalidad Muestra } n=332 \text { terceros } \\
\text { molares }\end{array}$} & \multirow{2}{*}{$\begin{array}{c}\text { Total de } \\
\text { terceros } \\
\text { molares } \\
n=332\end{array}$} \\
\hline & $\begin{array}{c}\text { Maxila } \\
\mathrm{n}=83\end{array}$ & $\begin{array}{l}\text { Mandíbula } \\
\mathrm{n}=82\end{array}$ & $\begin{array}{c}\text { Maxila } \\
\mathrm{n}=85\end{array}$ & $\begin{array}{c}\text { Mandíbula } \\
\mathrm{N}=82\end{array}$ & $\begin{array}{l}\text { Maxila } \\
\mathrm{n}=168\end{array}$ & $\begin{array}{c}\text { Mandíbula } \\
\mathrm{n}=164\end{array}$ & \\
\hline $\begin{array}{l}\text { Erupcionado } \\
\text { (nivel A) }\end{array}$ & $17 / 83=20,5 \%$ & $17 / 82=20,7 \%$ & $22 / 85=25,9 \%$ & $21 / 82=25,6 \%$ & $39 / 168=23,2 \%$ & $37 / 164=22,5 \%$ & $\begin{array}{l}76 / 332 \\
22,9 \%\end{array}$ \\
\hline $\begin{array}{l}\text { Semiretenido } \\
\text { (nivel B) }\end{array}$ & $22 / 83=26,5 \%$ & $30 / 82=36,6 \%$ & $23 / 85=27,0 \%$ & $21 / 82=25,6 \%$ & $45 / 168=26,8 \%$ & $48 / 164=29,3 \%$ & $\begin{array}{c}93 / 332 \\
28 \%\end{array}$ \\
\hline Retención (nivel C) & $44 / 83=53,0 \%{ }^{1}$ & $35 / 82=42,7 \%{ }^{1}$ & $40 / 85=47,1 \%{ }^{2}$ & $40 / 82=48,8 \%{ }^{2}$ & $84 / 168=50,0 \% \%^{3}$ & $79 / 164=48,2 \%{ }^{3}$ & $\begin{array}{l}163 / 332 \\
49,1 \%\end{array}$ \\
\hline Total & $83=100 \%$ & $82=100 \%$ & $85=100 \%$ & $82=100 \%$ & $168=100 \%$ & $164=100 \%$ & $\begin{array}{c}332 \\
100 \%\end{array}$ \\
\hline Agenesia & $17 / 100=17 \%$ & $18 / 100=18 \%$ & $15 / 100=15 \%$ & $18 / 100=18 \%$ & $32 / 200=16 \%$ & $36 / 200=18 \%$ & \\
\hline
\end{tabular}

La formación dentaria, según clasificación de Nolla, muestra un promedio de 9,05 DS 1,3 para 3Ms superiores y 8,75 DS 1,5 para los $3 \mathrm{Ms}$ inferiores, lo que demuestra una formación radicular más completa a nivel de maxilares, ratificando lo descrito en la literatura para la edad del grupo en estudio.
El porcentaje obtenido de retención del 3M fue de $49,1 \%$, con diferencia estadística significativa al $95 \%$ respecto de 3Ms erupcionados o semierupcionados (Tablas III, V y VIII), detectando similitud de porcentajes con investigadores latino americanos. Pese a no existir diferencias estadísticas significativas entre arcadas de individuos del mis- 
Tabla VI. Clasificación de Winter de terceros molares retenidos. Mujeres, hombres y total de muestra, de jóvenes de 17-20 años de edad, Antofagasta, Chile ( $\mathrm{n}=100)$.

\begin{tabular}{|c|c|c|c|c|c|c|c|c|c|c|c|c|}
\hline \multirow{2}{*}{$\begin{array}{l}\text { Clasificación Winter } \\
\text { Posición }\end{array}$} & \multicolumn{4}{|c|}{ Mujeres $n=83$ molares retenidos } & \multicolumn{4}{|c|}{ Hombres $\mathrm{n}=\mathbf{8 0} \mathbf{~ m o l}$ ares retenidos } & \multicolumn{4}{|c|}{$\begin{array}{c}\text { Total muestra n=163 mola res } \\
\text { retenidos }\end{array}$} \\
\hline & $\begin{array}{c}1.8 \\
\mathrm{n}=24\end{array}$ & $\begin{array}{c}2.8 \\
\mathrm{n}=20\end{array}$ & $\begin{array}{c}3.8 \\
\mathrm{n}=18\end{array}$ & $\begin{array}{c}4.8 \\
\mathrm{n}=21\end{array}$ & $\begin{array}{c}1.8 \\
\mathrm{~N}=22\end{array}$ & $\begin{array}{c}2.8 \\
\mathrm{n}=18\end{array}$ & $\begin{array}{c}3.8 \\
\mathrm{n}=22\end{array}$ & $\begin{array}{c}4.8 \\
\mathrm{n}=18\end{array}$ & $\begin{array}{c}1.8 \\
\mathrm{~N}=46\end{array}$ & $\begin{array}{c}2.8 \\
\mathrm{n}=38\end{array}$ & $\begin{array}{c}3.8 \\
\mathrm{n}=40\end{array}$ & $\begin{array}{c}4.8 \\
\mathrm{n}=39\end{array}$ \\
\hline Vertical & $\begin{array}{c}10 \\
41,7 \%\end{array}$ & $\begin{array}{c}10 \\
50,0 \%\end{array}$ & $\begin{array}{c}1 \\
5,6 \%\end{array}$ & $\begin{array}{c}1 \\
4,8 \%\end{array}$ & $\begin{array}{c}17 \\
77,3 \%\end{array}$ & $\begin{array}{c}13 \\
72,2 \%\end{array}$ & $\begin{array}{c}1 \\
4,5 \%\end{array}$ & 0 & $\begin{array}{c}27 \\
58,7 \%\end{array}$ & $\begin{array}{c}23 \\
60,5 \%\end{array}$ & $\begin{array}{c}2 \\
5,0 \%\end{array}$ & $\begin{array}{c}1 \\
2,5 \%\end{array}$ \\
\hline Mesio angulado & $\begin{array}{c}9 \\
37,5 \%\end{array}$ & $\begin{array}{c}3 \\
15,0 \%\end{array}$ & $\begin{array}{c}11 \\
61,1 \%\end{array}$ & $\begin{array}{c}15 \\
71,4 \%\end{array}$ & $\begin{array}{c}3 \\
13,6 \%\end{array}$ & $\begin{array}{c}2 \\
11,1 \%\end{array}$ & $\begin{array}{c}12 \\
54,6 \%\end{array}$ & $\begin{array}{c}14 \\
77,8 \%\end{array}$ & $\begin{array}{c}12 \\
26,1 \%\end{array}$ & $\begin{array}{c}5 \\
13,2 \%\end{array}$ & $\begin{array}{c}23 \\
57,5 \%\end{array}$ & $\begin{array}{c}29 \\
74,4 \%\end{array}$ \\
\hline Disto angulado & $\begin{array}{c}5 \\
20,8 \%\end{array}$ & $\begin{array}{c}7 \\
35,0 \%\end{array}$ & 0 & 0 & $\begin{array}{c}2 \\
9,1 \%\end{array}$ & $\begin{array}{c}3 \\
16,7 \%\end{array}$ & 0 & 0 & $\begin{array}{c}7 \\
15,2 \%\end{array}$ & $\begin{array}{c}10 \\
26,3 \%\end{array}$ & 0 & 0 \\
\hline Horizontal & & & $\begin{array}{c}4 \\
22,2 \%\end{array}$ & $\begin{array}{c}1 \\
4,8 \%\end{array}$ & & & $\begin{array}{c}8 \\
36,4 \%\end{array}$ & $\begin{array}{c}4 \\
22,2 \%\end{array}$ & & & $\begin{array}{c}12 \\
30,0 \%\end{array}$ & $\begin{array}{c}5 \\
12,8 \%\end{array}$ \\
\hline Vestíbulo lingual & & & $\begin{array}{c}2 \\
11,1 \%\end{array}$ & $\begin{array}{c}4 \\
19,0 \%\end{array}$ & & & $\begin{array}{c}1 \\
4,5 \%\end{array}$ & 0 & & & $\begin{array}{c}3 \\
7,5 \%\end{array}$ & $\begin{array}{c}4 \\
10,3 \%\end{array}$ \\
\hline
\end{tabular}

Tabla VII. Clasificación de Winter de terceros molares retenidos por arcada. Mujeres, hombres y total de muestra, de jóvenes de 17-20 años de edad, Antofagasta, Chile ( $\mathrm{n}=100)$. (1) $\mathrm{p}<1,02141 \mathrm{E}-14$, con diferencia estadística significativa al $95 \%$ entre terceros molares retenidos en la maxila en posición vertical respecto retención vertical en mandíbula para la totalidad de la muestra. (2) p<2,61306E-9 con diferencia estadística significativa al $95 \%$ entre terceros molares retenidos en la mandíbula en posición mesioangulada respecto de retención mesioangulada en maxila para la totalidad de la muestra. (3) p $<0,00000412757$, con diferencia estadística significativa al 95\% entre terceros molares retenidos en la mandíbula en posición mesio angulada respecto de las otras posiciones de retención en el género femenino. (4) p<0,0017221, con diferencia estadística significativa al 95\% entre terceros molares retenidos en la mandíbula en posición mesio angulada respecto de las otras posiciones de retención en el género masculino.

\begin{tabular}{|c|c|c|c|c|c|c|c|c|c|c|c|c|}
\hline \multirow{2}{*}{$\begin{array}{l}\text { Clasificación Winter } \\
\text { Posición }\end{array}$} & \multicolumn{4}{|c|}{$\begin{array}{c}\text { Mujeres } \\
\mathrm{n}=\mathbf{8 3} \text { molares retenidos }\end{array}$} & \multicolumn{4}{|c|}{$\begin{array}{c}\text { Hombres } \\
\mathbf{n}=\mathbf{8 0} \text { molares retenidos }\end{array}$} & \multicolumn{4}{|c|}{$\begin{array}{c}\text { Total muestra } \\
\text { n=163 molares retenidos }\end{array}$} \\
\hline & $\begin{array}{c}1.8 \\
\mathrm{n}=24\end{array}$ & $\begin{array}{c}2.8 \\
\mathrm{n}=20\end{array}$ & $\begin{array}{c}3.8 \\
\mathrm{n}=18\end{array}$ & $\begin{array}{c}4.8 \\
\mathrm{n}=21\end{array}$ & $\begin{array}{c}1.8 \\
\mathrm{~N}=22\end{array}$ & $\begin{array}{c}2.8 \\
\mathrm{n}=18\end{array}$ & $\begin{array}{c}3.8 \\
\mathrm{n}=22\end{array}$ & $\begin{array}{c}4.8 \\
\mathrm{n}=18\end{array}$ & $\begin{array}{c}1.8 \\
\mathrm{~N}=46\end{array}$ & $\begin{array}{c}2.8 \\
\mathrm{n}=38\end{array}$ & $\begin{array}{c}3.8 \\
\mathrm{n}=40\end{array}$ & $\begin{array}{c}4.8 \\
\mathrm{n}=39\end{array}$ \\
\hline Vertical & $\begin{array}{c}10 \\
41,7 \%\end{array}$ & $\begin{array}{c}10 \\
50,0 \%\end{array}$ & $\begin{array}{c}1 \\
5,6 \%\end{array}$ & $\begin{array}{c}1 \\
4,8 \%\end{array}$ & $\begin{array}{c}17 \\
77,3 \%\end{array}$ & $\begin{array}{c}13 \\
72,2 \%\end{array}$ & $\begin{array}{c}1 \\
4,5 \%\end{array}$ & 0 & $\begin{array}{c}27 \\
58,7 \%\end{array}$ & $\begin{array}{c}23 \\
60,5 \%\end{array}$ & $\begin{array}{c}2 \\
5,0 \%\end{array}$ & $\begin{array}{c}1 \\
2,5 \%\end{array}$ \\
\hline Mesio angulado & $\begin{array}{c}9 \\
37,5 \%\end{array}$ & $\begin{array}{c}3 \\
15,0 \%\end{array}$ & $\begin{array}{c}11 \\
61,1 \%\end{array}$ & $\begin{array}{c}15 \\
71,4 \%\end{array}$ & $\begin{array}{c}3 \\
13,6 \%\end{array}$ & $\stackrel{2}{11,1 \%}$ & $\begin{array}{c}12 \\
54,6 \%\end{array}$ & $\begin{array}{c}14 \\
77,8 \%\end{array}$ & $\begin{array}{c}12 \\
26,1 \%\end{array}$ & $\begin{array}{c}5 \\
13,2 \%\end{array}$ & $\begin{array}{c}23 \\
57,5 \%\end{array}$ & $\begin{array}{c}29 \\
74,4 \%\end{array}$ \\
\hline Disto angulado & $\begin{array}{c}5 \\
20,8 \%\end{array}$ & $\begin{array}{c}7 \\
35,0 \%\end{array}$ & 0 & 0 & $\begin{array}{c}2 \\
9,1 \%\end{array}$ & $\begin{array}{c}3 \\
16,7 \%\end{array}$ & 0 & 0 & $\begin{array}{c}7 \\
15,2 \%\end{array}$ & $\begin{array}{c}10 \\
26,3 \%\end{array}$ & 0 & 0 \\
\hline Horizontal & & & $\begin{array}{c}4 \\
22,2 \%\end{array}$ & $\begin{array}{c}1 \\
4,8 \%\end{array}$ & & & $\begin{array}{c}8 \\
36,4 \%\end{array}$ & $\begin{array}{c}4 \\
22,2 \%\end{array}$ & & & $\begin{array}{c}12 \\
30,0 \%\end{array}$ & $\begin{array}{c}5 \\
12,8 \%\end{array}$ \\
\hline Vestíbulo lingual & & & $\begin{array}{c}2 \\
11,1 \%\end{array}$ & $\begin{array}{c}4 \\
19,0 \%\end{array}$ & & & $\begin{array}{c}1 \\
4,5 \%\end{array}$ & 0 & & & $\begin{array}{c}3 \\
7,5 \%\end{array}$ & $\begin{array}{c}4 \\
10,3 \%\end{array}$ \\
\hline
\end{tabular}

mo género, así como entre mujeres y hombres, predomina la retención maxilar en el género femenino $(53,0 \%)$ y en toda la muestra $(50,0 \%)$. Al analizar la relación del $3 \mathrm{M}$ con la rama mandibular, se detecta $25 \%$ de cordales de Clase III.

Respecto a la clasificación de Winter (ver Tablas IV, VI. y VII), en la muestra total predomina la impactación mesioangulada $(42,3 \%)$ coincidente con la mayoría de los resultados revisados en la literatura (Tabla VIII). Los 3Ms maxilares retenidos presentan un 59,6\% de retención vertical, con diferencia estadística significativa al $95 \%$ $(\mathrm{p}<1,02141 \mathrm{E}-14)$ respecto de retención vertical mandibular. En la mandíbula, para toda la muestra se aprecia un porcentaje de $65,8 \%$ de dientes mesioangulados con un diferencia estadística significativa al $95 \%$ respecto de la posición mesioangulada en maxila ( $\mathrm{p}<2,61306 \mathrm{E}-9)$, con similares resultados en mujeres con un $66,7 \%(\mathrm{p}<0,000323695)$ y en hombres con un 65,0\% ( $\mathrm{p}<0,0000144241)$.
La Tabla VIII permite comparar nuestros resultados con porcentajes de retención y posición determinados por diversos autores, calculándose $\mathrm{p}<0,05$ para significancia estadística, siempre y cuando se tuviese acceso a los datos necesarios para poder realizar la comparación estadística.

El análisis comparativo de la Tabla VIII, establece que existen estudios que coinciden con nuestros resultados y sin diferencia estadística de significancia al 95\% (retención total: Nicodemo Filho et al., 2007; Cienfuegos et al., 2008; en mandíbula Hattab, 1997). En cambio, se muestran diferencias estadísticas significativas al $95 \%$ en relación a nuestros resultados de retención total: Queck et al. (2003); Sandhu \& Kaur; Susarla \& Dodson (2004); Susarla \& Dodson (2005); Rodríguez et al. (2007); en maxila: Chaparro-Avendaño et al.; Queck et al.; Mateos \& Hernández (2005) y en mandíbula Hattab et al. (1995); Llerena \& Arrascue (2006); Martínez et al.; Queck et al. y Mateos \& Hernández. 
La retención en posición disto angulada predomina en forma significativa en $3 \mathrm{Ms}$ maxilares respecto de $3 \mathrm{Ms}$ mandibulares $(p<0,0000243969)$. Esta posición de retención es considerada por varios autores como predisponente a complicaciones operatorias y postoperatorias en la exodoncia del $3 \mathrm{M}$.
La retención horizontal se aprecia sólo a nivel mandibular correspondiendo al segundo tipo de retención con alto porcentaje de casos $(21,5 \%$ en toda la muestra; $12,8 \%$ en mujeres y $30 \%$ en hombres). Algunos $3 \mathrm{Ms}$ en posición horizontal tenían las cúspides distales semierupcionadas (posición B de Pell-Gregory) y producto

Tabla VIII. Porcentaje de posición de terceros molares retenidos obtenidos por diversos autores según, la Clasificación de Winter y comparación $\mathrm{p}<0,05$ respecto del porcentaje de retención determinado en la investigación $(49,1 \%)$.

\begin{tabular}{|c|c|c|c|c|c|c|c|c|c|}
\hline In vestigad or & $\begin{array}{c}\text { Edad } \\
\text { Rango }\end{array}$ & $\begin{array}{l}\mathbf{V} \\
\%\end{array}$ & $\underset{\%}{\mathrm{MA}}$ & $\begin{array}{l}\text { DA } \\
\%\end{array}$ & $\begin{array}{c}\text { H y otras } \\
\%\end{array}$ & $\mathbf{n}^{\circ} \mathbf{3 M}$ & $\mathbf{n}^{\circ}$ casos & $\begin{array}{c}\text { Retención } \\
\%\end{array}$ & $\mathrm{p}<0,05$ \\
\hline García et al., 2009 & 18,6 & 32,5 & 42,3 & 10,4 & 14,7 & 332 & 100 & 49,1 (total) & \\
\hline Nicodemo et al., 1982 & $17-20$ & $\begin{array}{c}44,5 \max \\
44,5 \\
\text { mand }\end{array}$ & $\begin{array}{c}52,0 \text { max } \\
56,2 \\
\text { mand }\end{array}$ & & & & & $\begin{array}{c}50 \mathrm{ma}-48,2 \mathrm{md} \\
40,0 \text { (total) }\end{array}$ & \\
\hline Martínez et al., 1995 & $\begin{array}{c}22,3 \\
18-25\end{array}$ & 40,3 & 50,6 & 4,3 & 4,8 & 186 & 116 & $37,6 \mathrm{~m}$ and & $\begin{array}{c}0,01160 \\
14\end{array}$ \\
\hline Hattab et al., 1995 & 20,4 & 41,0 & 50,0 & & & 844 & 232 & 26,6 (total) & $\begin{array}{c}1,49214 \\
\text { E-13 }\end{array}$ \\
\hline Hattab, 1997 & $\begin{array}{c}19,7 \\
18,6-20,8\end{array}$ & & & & & 67 mand & 36 & $46,0 \mathrm{~m}$ and & $\begin{array}{c}0,76125 \\
8\end{array}$ \\
\hline Hattab et al., 1999 & $\begin{array}{c}19,8 \\
18,5- \\
20.8\end{array}$ & & & & & 213 mand & 134 & $17,4 \mathrm{~m}$ and & $\begin{array}{c}1,35137 \\
\text { E-10 }\end{array}$ \\
\hline Punwutikorn, et al., 1999 & $13-69$ & 26,9 & 39,9 & 13,0 & 20,2 & 1151 & & 16,7 mand & $\mathbf{0 , 0}$ \\
\hline Kruger et al., 2001 & 18 & $\begin{array}{c}18,1 \max \\
11,9 \\
\text { mand }\end{array}$ & $\begin{array}{c}29,3 \text { max } \\
62,9 \\
\text { mand }\end{array}$ & $\begin{array}{c}12,2 \\
\max \\
1,4 \\
\text { mand }\end{array}$ & $\begin{array}{c}0,0 \text { max } \\
1,0 \text { mand }\end{array}$ & $\begin{array}{c}1374 \text { max } \\
1278 \\
\text { mand }\end{array}$ & 821 & & \\
\hline Renton et al., 2001 & & 28,0 & 37,0 & 22,0 & 13,0 & 354 mand & 354 & & \\
\hline Queck et al., 2003 & 26,5 & 9,5 & 59,5 & 9,8 & $\begin{array}{c}21,2 \\
\mathrm{H}=18,8\end{array}$ & 2281 & 686 & $\begin{array}{c}15,0 \text { (total) } \\
39 \text { max - } 9 \text { md }\end{array}$ & $\begin{array}{c}\mathbf{0 , 0} \\
\mathbf{0 , 0 2 0 6} \\
-\mathbf{0 , 0}\end{array}$ \\
\hline Susarla \& Dodson, 2004 & $\begin{array}{c}26,2 \\
15-65\end{array}$ & 68,4 & 20,0 & 6,8 & 4,8 & 250 & 82 & 15,0 (total) & $\mathbf{0 , 0}$ \\
\hline Sandhu \& Kaur, 2005 & $\begin{array}{c}19,3 \\
17,5-20\end{array}$ & $\begin{array}{c}43,0 \text { max } \\
42,0 \\
\text { mand }\end{array}$ & $\begin{array}{c}9,0 \text { max } \\
49,0 \\
\text { mand }\end{array}$ & $\begin{array}{c}48,0 \\
\max \\
9,0 \\
\text { mand }\end{array}$ & & 354 & 100 & $\begin{array}{c}34,0 \text { (total) } \\
59 \text { max-10 md }\end{array}$ & $\begin{array}{c}0,00004 \\
169\end{array}$ \\
\hline Susarla \& Dodson, 2005 & $\begin{array}{c}26,6 \\
15-65\end{array}$ & 64,0 & 22,8 & 8,4 & 4.8 & 450 & 150 & 15,0 (total) & $\mathbf{0 , 0}$ \\
\hline Susarla \& Dodson, $2005^{\mathrm{b}}$ & $\begin{array}{c}25,6 \\
14-65\end{array}$ & 61,6 & 24,0 & 9,8 & 4,7 & 253 & 150 & 15,6 (total) & $\mathbf{0 , 0}$ \\
\hline Chaparro et al., 2005 & $12-18$ & $\begin{array}{c}80,0 \max \\
27,0 \\
\text { mand }\end{array}$ & $\begin{array}{c}13,3 \text { max } \\
71,5 \\
\text { mand }\end{array}$ & $\begin{array}{c}6,7 \max \\
1,5 \\
\text { mand }\end{array}$ & & $\begin{array}{l}120 \\
270\end{array}$ & 173 & $\begin{array}{c}75,0 \text { max } \\
46,7 \text { mand }\end{array}$ & $\begin{array}{c}\mathbf{0 , 0 0 0 0 1} \\
\mathbf{8 9 4} \\
0,55774 \\
9\end{array}$ \\
\hline Mateos et al., 2005 & $\begin{array}{l}30,76 \\
17-72\end{array}$ & 31,13 & 49,28 & 7,69 & 11,90 & $\begin{array}{c}279 / 5730 \\
1118 / 573 \\
0\end{array}$ & 2865 & $\begin{array}{c}\text { 4,9 max } \\
19,5 \text { mand }\end{array}$ & $\begin{array}{l}\mathbf{0 , 0} \\
\mathbf{0 , 0}\end{array}$ \\
\hline Llerena \& Arrascue, 2006 & $\begin{array}{c}24 \\
15-56\end{array}$ & 29,0 & 55,0 & 9,0 & 7,0 & 100 mand & & 5,0 mand & $\begin{array}{c}2,70894 \\
\text { E-13 }\end{array}$ \\
\hline Nicodemo et al. 2007 & $18-35$ & 59,5 & 35,1 & 5,4 & 2,0 & 164 & 41 & 46,0 (total) & $\begin{array}{c}0,70791 \\
3\end{array}$ \\
\hline Rodríguez, et al., 2007 & $\begin{array}{c}20,6- \\
21,2 \\
11-72\end{array}$ & 43,9 & 40,8 & 6,1 & 9,2 & 2120 & 1072 & 41,6 (total) & $\begin{array}{c}0,01018 \\
31\end{array}$ \\
\hline Cienfuegos et al., 2008 & 29,8 & 45,27 & 24,88 & 15,42 & 14,43 & 201 & 115 & 43,7 & $\begin{array}{c}0,21878 \\
9\end{array}$ \\
\hline
\end{tabular}


del impacto distal al 2M que impide cualquier posibilidad de erupción normal, estos dientes fueron clasificados como retenidos (posición C de Pell-Gregory). Los 3Ms retenidos en posición vestíbulolingual, o a la inversa, se visualizan solamente en mandíbula ( $8,9 \%$ de los casos) y para el análisis estadístico fueron agrupados con la posición horizontal y otras.

\section{CONCLUSIONES}

Se determinó un 32\% de agenesia en la muestra de jóvenes entre 17-20 años de edad, con presencia de 332 $3 \mathrm{Ms}$ con formación dentaria tipo 9 de Nolla para 3Ms superiores y entre 8-9 para 3Ms mandibulares.

La retención total fue de $49,1 \%$ con diferencia estadística siginificativa al $95 \%$ respecto de dientes erupcionados o semierupcionados, sin diferencias entre ambas arcadas según género y también entre hombres y mujeres. Se determinó un 25\% de 3Ms Clase III respecto de la rama mandibular. La literatura muestra variedad de resultados comparativos, con y sin significancia estadística $(\mathrm{p}<0,05)$, en relación a nuestros datos de retención.

Coincidente con la literatura predominó la retención mesioangulada, a continuación la posición vertical y finalmente, posiciones distoangulada y horizontal con porcentajes similares. En la retención maxilar predominó la posición vertical mientras que en la retención mandibular prevaleció la posición mesio angulada, comprobándose significativos porcentajes de retención horizontal y vestíbulo lingual o viceversa, tanto en la totalidad de la muestra como en ambos géneros. La retención disto angulada se produjo a nivel maxilar y fue considerada como predisponente para producir complicaciones postoperatorias.

De acuerdo al alto porcentaje de retención determinado en el grupo etáreo y teniendo en cuenta los costos clínicos, ausencias laborales y las complicaciones postquirúrgicas que acarrean las exodoncias profiláctica o preventiva de $3 \mathrm{Ms}$, creemos conveniente sugerir a los cirujanos retardar el mayor tiempo posible los procedimientos quirúrgicos con fines profilácticos, pensando en la probabilidad que se produza la erupción tardía, lo cual fue demostrado por Hattab, Ventä et al. (1999, 2004) y Kruger et al. (2001) quienes realizaron estudios longitdinales, observando disminución significativa de retención producto de erupciones durante el tiempo en que duró la investigación. Nuestra propuesta es avalada por el meta-análisis realizado por Haug et al. (2005) en 3760 pacientes de 25 años o mayores a los cuales se les realizó exodoncia quirúrgica del $3 \mathrm{M}$ y que detallan escasa morbilidad, con baja incidencia de complicaciones y mínimo impacto en la calidad de vida del paciente

\section{AGRADECIMIENTOS}

Los autores expresan sus agradecimientos a la administración y al personal de la Clínica de Ortodoncia Manquehue, Antofagasta, Chile por su colaboración y su ayuda para la realización del presente trabajo de investigación.

GARCÍA-HERNÁNDEZ, F.; TORO, Y. O.; VEGA, V. M. \& VERDEJO, M. M. Eruption and retention of third molars in young people between 17 and 20 years of age, Antofagasta, Chile. Int. J. Morphol., 27(3):727-736, 2009.

SUMMARY: Intraoseous retention and anomalous eruption position of $3 \mathrm{Ms}$ had been widely studied. They are probably produced by reducing the size of jaws by changes in eating habits, reducing the retro molar space, making it difficult normal eruption between 15 and 25 years old, producing pathologies or discomfort by compromising nearly orofacial structures. The above, has promoted the extraction or prophylactic orthodontic, even from the dental germ, with expensives clinical cost, hospital surgery, compromising part of the resources to finance other surgical oral procedures, in addition to risks during surgery, postoperative and iatrogenic injuries temporary or permanent. The sample consisted in 100 young people aged 17 to 20 years of age (50 women and 50 men) of the city of Antofagasta, healthy, without general and maxillofacial malformation, without having submitted infectious diseases that distort odontogenesis and periods of eruption, without extractions of 3M nor orthodontic treatments prior to the examination of the $\mathrm{x}$-ray overview and classifying eruption of 3Ms as tables of PellGregory and Winter. It was determined that $49.1 \%$ of $3 \mathrm{Ms}$ were retained, with $\mathrm{p}<0.05$ significant with regard to erupted teeth, predominate maxillary retention, especially in women. In all cases and in maxilla predominate $3 \mathrm{Ms}$ with mesiangular position ( $\mathrm{p}<0.05$ of significance) and in maxillary vertical retention ( $\mathrm{p}<0.05$ of significance). Prevailing $3 \mathrm{Ms}$ maxilla with distoangular position, a position that predisposes surgical complications and postoperative extractions. The horizontal retention is apparent only in mandible, being the second type of retention (21.5\% in all cases and $30 \%$ in men). Without a clinic decision to indicate surgery, we suggest to postpone the prophylactic extraction of $3 \mathrm{Ms}$, awaiting possible late eruption (Hattab, 1997; Ventä et al., 1999, 2004; Kruger et al., 2001). Meta-analyzes show minimum morbidity in 3Ms extractions in patients aged 25 or older, with low incidence of complications and minimum impact on quality of life (Haug et al., 2005).

KEY WORDS: Third molar; Retention; Pell-Gregory classification; Winter classification; Dentistry. 


\section{REFERENCIAS BIBLIOGRÁFICAS}

Ahlqwist, M. \& Gröndahl, H. G. Prevalence of impacted teeth and associated pathology in middle-age and older Swedish women. Community Dent. Oral Epidemiol., 19:116-9, 1991.

Arboleda, L. A.; Echeverri, J.; Restrepo, L. A.; Marín, M. L.; Vásquez, G.; Gómez, J. C.; Manco, H. A.; Pérez, C. M \& Taborda, E. Agenesia dental. Revisión bibliográfica y reporte de dos casos clínicos. Rev. Fac. Odontol. Univ. Antioq., 18(1):47-54, 2006.

Bailit, H. L. Dental variation among population. An anthropologic view. Dent. Clin. North Am., 19(1):125-39, 1975.

Björk, A. Mandibular growth and third molar impaction. Acta Odontol. Sacand., 14:231-6, 1956.

Cauvi, D. \& Feldman, I. Presencia clínica de los terceros molares y su relación con la pérdida de los primeros y/o segundos molares permanentes. Rev. Iberoamericana de Ort., 9(1):925,1989 .

Chaparro-Avendaño, A.; Pérez-García, S.; Valmaseda-Castellón, E.; Berini-Aytés, L. \& Gay-Escoda, C. Morbilidad de la extracción de los terceros molares entre los 12 y 18 años de edad. Med. Oral Patol. Oral Cir. Bucal., 10:422-31, 2005.

Cienfuegos, A. G; González, B \& Nava, T. Frecuencia de retención de terceros molares. Disponible en:http:// odontologia.iztacala.unam.mx/instrum_y_lab1/otros/ ColoquioXVI/contenido/indice_cartel_archivos/ TRAB\%20COMPL\%20COLOQ20CARTEL/HTML/ 1312.htm (fecha de acceso 26 de Septiembre de 2008).

Feldman, I.; Estupiñán, C.; Cauvi, D.; Espinoza, A. \& Rajecic, Z. Estudio comparado de agenesia de terceros molares en pacientes fisurados y no fisurados. Rev. Fac. Odont. Univ. de Chile, 13(1):26-35, 1995.

Figún, M. E. \& Garino, R. R. Anatomía odontológica funcional y aplicada. 2a Ed. Buenos Aires, El Ateneo, 1992. pp.341-2.

Flygare, L. \& Öhman, A. Preoperative imaging procedures for lower wisdom teeth removal. Clin. Oral Invest., 12:291$302,2008$.

Friedman, J. W. Containing the cost of third-molar extractions: a dilema for health insurance. Public Health Rep., 98(4):376-84, 1983.

Friedman, J. W. The prophylactic extraction of third molars: a public health hazard. Am. J. Public Health, 97(9):1554-9, 2007.
García-Hernández, F. \& Araneda, C. Agenesia del tercer molar en pacientes atendidos en la clínica odontológica de la Universidad de Antofagasta, Chile. Int. J. Morphol., 27(2):393-402, 2009

García-Hernández, F. \& Beltrán, V. Agenesia del tercer molar en una etnia originaria del Norte de Chile: Atacameños o lican antai. Int. J. Morphol., 26(3):583-90, 2008.

García-Hernández, F. \& Beltrán, V. Agenesia del tercer molar en una etnia originaria del Norte de Chile: Aymarás. Int. J. Morphol., 27(1):157-64, 2009.

García-Hernández, F.; Toro Y. O.; Vega, V. M. \& Verdejo M. M. Agenesia del tercer molar en jóvenes entre 14 y 20 años de edad, Antofagasta, Chile. Int. J. Morphol., 26(4):825-32, 2008.

Halmos, D. R.; Ellis, E. \& Dodson, T. B. Mandibular third molars and angle fracture. J. Oral Maxillofac. Surg., 62:1076-81, 2004.

Hattab, F. N. \& Alhaija, E. S. J. Radiographic evaluation of mandibular third molar eruption. Oral Surg. Oral Med. Oral Patol. Oral Radiol. Endod., 88:285-91, 1999.

Hattab, F. N. Positional changes and eruption of impacted mandibular third molars in young adults: A radiographic 4 year follow up study. Oral Surg. Oral Radiol. Endod., 84:604, 1997.

Hattab, F. N.; Rawashdeh, M. A. \& Fahmy, M. S. Impaction status of third molars in Jordanian students. Oral Surg. Oral Med. Oral Pathol. Oral Radiol. Endon., 79(1):24-9, 1995.

Haug, R. H; Perrott, D. H.; González, M. L. \& Talwar, R. M. The American Association of Oral and Maxillofacial Surgeons agerelated third molar study. J. Oral Maxillofac. Surg., 63:110614, 2005.

Kruger, E.; Thomson, W. M. \& Konthasinghe, P. Third molar outcomes from age 18 to 26 from a population-based New Zealand longitudinal study. Oral Surg. Oral Med. Oral Pathol. Oral Radiol. Endod., 92:150-5, 2001.

Llerena, G. \& Arrascue, M. Tiempo de cirugía efectiva en la extracción de terceros molares realizadas por un cirujano oral y maxilofacial con experiencia. Rev. Estomatol. Herediana, 16:(1):40-5, 2006.

Martínez, S.; Concha, G. \& San Pedro, J. Estudio radiográfico de terceros molares inferiores en una muestra de individuos de 18 a 20 años. Rev. Fac. Odont. Univ. de Chile, 13(1):43-9, 1995.

Mateos, I.. \& Hernández, F. Prevalencia de inclusión dental y patología asociada en pacientes de la Clínica de la Facultad de Odontología Mexicali de la UABC. Revista Odontológica Mexicana, 9(2):84-91, 2005. 
Ministerio de Salud de Chile. Reacciones adversas en atención dental. Departamento de Salud Bucal y Departamento de Estadística. www.redsalud.gov.cl/archivos/iih/dental/ 2006/pdf (fecha de acceso 02 de Noviembre de 2008).

Muhonen, A.; Ventä, I. \& Ylipaavalntemi, P. Factors predisposing postoperative complications related to wisdom tooth surgery among university students. J. Am. Coll. Health, 46:39-42, 1997.

Nicodemo Fhilo, R. A.; Rangel, F. J. C. \& Bazzarella, C. B. Prevalencia de terceiros molares inclusos entre estudantes da Facultade de Odontologia de Sâo José dos Campos. Ars. Cvrandi., 8(4):13-5, 1982.

Nicodemo Fhilo, R. A.; Louzada, J. \& Arisawa, E. A. L. Prevalência de terceiros molares inclusos entre estudantes de uma instituicão universitária. XI Encontro latino americano de iniciacão científica e VII Encontro latino americano de pós-graduacão. Universidade do Vale do Paraiba. 2007. http://www.inicepg.univap.br/INIC_07/trabalhos/ saude/inic/INICG00289_01C.pdf

Odusanya, S. A \& Abayomi, I. O. Third molar eruption among rural nigerians. Oral Surg. Oral Med. Oral Pathol., 71(2):151:4, 1991.

Olate, S.; Alister, J. P.; Alveal, R.; Thomas, D.; Soto, M.; Mancilla, P. \& Ceballos, M. Hallazgos clínicos y radiográficos de terceros molares con indicación de extracción. Resultados preliminares. Int. J. Odontoestomat., 1(1):29:34, 2007.

Pell, G. J. \& Gregory, G. T. Impacted third molars: Classification and modified technique for removal. Dent. Digest., 39:3308, 1933.

Punwutikorn, J.; Waikakul, A. \& Ochareon, P. Synptoms of unerupted mandibular third molar. Oral Surg. Oral Med. Oral Pathol. Oral Radiol. Endod., 87(3):305-10, 1999.

Quek, S. L.; Tay, C. K; Tay, K. H; Toh, S. L. \& Lim K. C. Pattern of third molar impaction in a Singapore Chinese population: A retrospective radiographic study. Int. J. Oral Maxillofac. Surg., 32:548-52, 2003.

Renton, T.; Smeeton, N. \& McGurk, M. Factors predictive of difficulty of mandibular third molar surgery. Br. Dent. J., 190(11):607-10, 2001.

Rodríguez, G. C.; Martínez, E.; Duque, F. L. \& Londoño, L. M. Caracterización de terceros molares sometidos a exodoncia quirúrgica en la Facultad de Odontología de la Universidad de Antioquia entre 1991 y 2001. Rev. Fac. Odontol. Univ. Antioquia, 18(2):76-83, 2007.
Sandhu S. \& Kaur, T. Radiographic evaluation of the status of the third molar in the Asian-Indian students. J. Oral Maxilofacial Surg., 63(5):640-5, 2005.

Sarmiento, P. \& Herrera, A. Agenesia de terceros molares en estudiantes de Odontología de la Universidad del Valle entre 16 y 25 años. Revista Colombia Médica, 35(3):5-9, 2004.

Shafer, W.; Hine, M. \& Levy, B. A textbook of Oral Pathology. $4^{\text {th }}$ Ed. Philadelphia, W.B. Saunders Company, 1977.

Silvestri Jr., A. R. \& Sing, I. The unresolved problem of the third molar: would people be better off without it? J. Am. Dent. Assoc., 134:450-5, 2003.

Susarla, S. M \& Dodson, T. B. Estimating third molar extraction difficulty: A comparison of subjetive and objetive factors. J. Oral Maxillofac. Surg., 63:427-34, 2005a.

Susarla, S. M \& Dodson, T. B. How well do clinicians estimate third molar extraction difficulty? J. Oral Maxillofac. Surg., 63:191-9, 2005b.

Susarla, S. M \& Dodson, T. B. Risk factors for third molarextraction difficulty. J. Oral Maxillofac. Surg., 62:1363-71, 2004.

Ventä, I.; Turtola, L. \& Ylippanvalniemi, P. Changes in clinical status of third molars in adults during 12 years of observation. J. Oral Maxillofac. Surg., 57:386-9, 1999.

Ventä, I.; Turtola, L. \& Ylippanvalniemi, P. Clinical follow up study of third molar eruption from ages 20 to 26 years. Oral Surg. Oral Med. Oral Pathol., 72:150, 1991.

Ventä, I.; Ylippanvalniemi, P. \& Turtola, L. Clinical outcomes of third molar in adults followed during 18 years. J. Oral Maxillofac. Surg., 62:182-5, 2004.

Winter, G. B. Principles of exodontia as applied to the impacted third molar. St. Louis, American Medical Books, 1926.

Dirección para correspondencia:

Prof. Dr. Fernando García-Hernández.

Unidad de Anatomía, Departamento Biomédico

Facultad de Ciencias de la Salud

Universidad de Antofagasta.

Coquimbo 945, Depto 64

Antofagasta

CHILE

Recibido : 22-06-2009

Aceptado: 24-07-2009 